Atomic Oxygen Abundance in Molecular Clouds:

Absorption Toward Sagittarius B2

D.C. Lis, J. Keene, T.G. Phillips, P. Schilke,

M.W. Werner \& J. Zmuidzinas

2001-7

To Appear in

The Astrophysical Journal 



\title{
Atomic Oxygen Abundance in Molecular Clouds: Absorption Toward Sagittarius B2
}

\author{
D.C. Lis $^{1}$, Jocelyn Keene ${ }^{1,2}$, T.G. Phillips ${ }^{1}$, P. Schilke ${ }^{3}$, M.W. Werner ${ }^{2}$, and J. Zmuidzinas ${ }^{1}$
}

\begin{abstract}
We have obtained high-resolution $\left(\sim 35 \mathrm{~km} \mathrm{~s}^{-1}\right)$ spectra toward the molecular cloud Sgr B2 at $63 \mu \mathrm{m}$, the wavelength of the ground-state fine-structure line of atomic oxygen (O1), using the ISO-LWS instrument. Four separate velocity components are seen in the deconvolved spectrum, in absorption against the dust continuum emission of Sgr B2. Three of these components, corresponding to foreground clouds, are used to study the OI content of the cool molecular gas along the line of sight. In principle, the atomic oxygen that produces a particular velocity component could exist in any, or all, of three physically distinct regions: inside a dense molecular cloud, in the UV illuminated surface layer (PDR) of a cloud, and in an atomic (HI) gas halo. For each of the three foreground clouds, we estimate, and subtract from the observed OI column density, the oxygen content of the $\mathrm{HI}$ halo gas, by scaling from a published high-resolution $21 \mathrm{~cm}$ spectrum. We find that the remaining OI column density is correlated with the observed ${ }^{13} \mathrm{CO}$ column density. From the slope of this correlation, an average $[\mathrm{Or}] /\left[{ }^{13} \mathrm{CO}\right]$ ratio of $270 \pm 120(3 \sigma)$ is derived, which corresponds to $[\mathrm{OI}] /[\mathrm{CO}] \approx 9$ for a $\mathrm{CO}$ to ${ }^{13} \mathrm{CO}$ abundance ratio of 30 . Assuming $a^{13} \mathrm{CO}$ abundance of $1 \times 10^{-6}$ with respect to $\mathrm{H}$ nuclei, we derive an atomic oxygen abundance of $2.7 \times 10^{-4}$ in the dense gas phase, corresponding to a $15 \%$ oxygen depletion compared to the diffuse ISM in our Galactic neighborhood. The presence of multiple, spectrally resolved velocity components in the Sgr B2 absorption spectrum allows, for the first time, a direct determination of the PDR contribution to the OI column density. The PDR regions should contain OI but not ${ }^{13} \mathrm{CO}$, and would thus be expected to produce an offset in the $\mathrm{OI}-{ }^{13} \mathrm{CO}$ correlation. Our data do not show such an offset, suggesting that within our beam OI is spatially coexistent with the molecular gas, as traced by ${ }^{13} \mathrm{CO}$. This may be a result of the inhomogeneous nature of the clouds.
\end{abstract}

Subject headings: Subject headings: infrared: ISM: lines and bands - ISM: abundances

\section{Introduction}

A longstanding problem for our understanding of the quiescent dense interstellar medium (ISM), often described as molecular clouds, has been the difficulty of accounting for the gas phase abundance of carbon and oxygen. Since the first calculations of ion-molecule schemes (Herbst \&

\footnotetext{
${ }^{1}$ California Institute of Technology, Downs Laboratory of Physics 320-47, Pasadena, CA 91125

2 Jet Propulsion Laboratory 264-767, Pasadena, CA 91109

${ }^{3}$ Max-Planck-Institut für Radioastronomie, Auf dem Hügel 69, D-53121 Bonn, Germany
}

Klemperer 1973; Dalgarno \& Black 1976) there have been theoretical predictions indicating that the fundamental reservoirs of these elements are the molecular species $\mathrm{CO}, \mathrm{O}_{2}$, and $\mathrm{H}_{2} \mathrm{O}$. It is usually assumed that the local gas phase oxygen abundance is about twice that of carbon. This comes from the observed stellar values, modified by the depletion seen in local ISM diffuse clouds such as those toward $\zeta$ Ophiuchi and HD154368 (Snow and Witt 1996; Snow et al. 1996; Cardelli et al. 1993), i.e. the carbon abundance $[C]=$ $1.66 \times 10^{-4}$ and $1.32 \times 10^{-4}$, respectively, and the oxygen abundance $[\mathrm{O}]=2.88 \times 10^{-4}$, giving an average $[\mathrm{C}] /[\mathrm{O}]=0.51$. So nearly all car- 
bon should be in $\mathrm{CO}$, with plenty of oxygen left over for $\mathrm{O}_{2}$ and $\mathrm{H}_{2} \mathrm{O}$. Millimeter-wave astronomy measurements have indeed shown the strong presence of $\mathrm{CO}$ at about $10^{-4}$ of $\mathrm{H}_{2}$ (e.g. Lada et al. 1994). However, the $\mathrm{H}_{2} \mathrm{O}$ abundance is only about $10^{-5}-10^{-7}$ of $\mathrm{H}_{2}$ (Jacq et al. 1988; Zmuidzinas et al. 1995; Cernicharo et al. 1997), or even lower, $10^{-7}-10^{-10}$, as measured by SWAS (Snell et al. 2000a, 2000b), except for strongly shocked gas, where it reaches $3.5 \times 10^{-4}$ (Melnick et al. 2000). For $\mathrm{O}_{2}$, only upper limits of $<10^{-5}$ of $\mathrm{H}_{2}$ (Liszt \& Vanden Bout 1985; Fuente et al. 1993), $(0.33 \pm 1.6) \times 10^{-7}$ (Goldsmith et al. 2000) are found. This may be compared with measurements of the $\mathrm{H}_{3} \mathrm{O}^{+}$ion abundance (Phillips et al. 1992) in the range of $10^{-9}-10^{-10}$ of $\mathrm{H}_{2}$, which lead to predictions of $\mathrm{H}_{2} \mathrm{O}$ abundances in the range $10^{-6}-10^{-7}$ and $\mathrm{O}_{2}$ in the range $10^{-5}-10^{-6}$. The observational status is consistent in that, after $\mathrm{CO}$, the anticipated major oxygen molecular species, $\mathrm{H}_{2} \mathrm{O}$ and $\mathrm{O}_{2}$, are deficient by at least an order of magnitude (see van Dishoeck et al. 1993 for a review).

It is known that some oxygen is tied up in $\mathrm{H}_{2} \mathrm{O}$ mantles on grains in the dense molecular clouds and this is sometimes put forward to suggest a $[\mathrm{C}] /[\mathrm{O}]$ ratio in the gas larger than standard. Whittet (1992) estimates from $3 \mu \mathrm{m}$ features that $N\left(\mathrm{H}_{2} \mathrm{O}\right) / N(\mathrm{H}) \simeq 8.6 \times 10^{-5}$ on grains in the Taurus dark cloud, and Knacke \& Larson (1991) find a factor of 10 less $\mathrm{H}_{2} \mathrm{O}$ in the gas phase on the lineof-sight toward Orion BN. There is then a possibility that a quarter of the oxygen budget is lost to grain mantles in high column density clouds. However, the clouds investigated here are on average $A_{v} \sim 1-10$ (see Table 1), which is somewhere between the values for the diffuse clouds in which the $\mathrm{C}$ and $\mathrm{O}$ abundances are directly measured, and the very high values at which $\mathrm{H}_{2} \mathrm{O}$ mantles are seen. Thus it seems likely that the traditional assumption of $[\mathrm{C}] /[\mathrm{O}] \sim 0.5$ in the gas phase is suitable here, with the uncertainties limited to about $25 \%$, at least as is known from the effect of $\mathrm{H}_{2} \mathrm{O}$ grain mantles.

In 1994 we proposed to use the ISO-LWS instrument to test whether the missing oxygen component was present in atomic form by means of absorption line spectroscopy of OI at $63 \mu \mathrm{m}$. Indeed, atomic oxygen is predicted by chemical models to be present in the dense ISM (see e.g. van Dishoeck et al. 1993; as well as Bergin et al. 2000 and references therein for a discussion of recent chemical models). Thus purely on the basis of ion-molecule models of ISM chemistry, there is a strong argument for searching for Ol absorption in cool, shielded clouds, as a fundamental constituent.

However, the situation is complex. Molecules near the edges of clouds are known to be photodissociated by interstellar UV photons (e.g. Langer 1976; Hollenbach et al. 1991). These photon dominated regions (PDRs) may show strong Ol emission (Melnick et al. 1979), Cil emission (Russell et al. 1980), and Cr emission (Phillips \& Huggins 1981). In the case of $\mathrm{CI}$, where relatively high spectral and spatial resolution is available, it has proved quite hard to separate the individual contributions of the PDR and the quiescent dense cloud (Keene et al. 1985, 1997). One difficulty, then, is to distinguish between the PDR and quiescent dense medium contributions to the atomic oxygen column density. The distribution of atomic oxygen probably extends deeper into the cloud than that of atomic carbon, because the dissociation threshold for $\mathrm{O}_{2}$ (about $5.1 \mathrm{eV}$ ) is considerably less than for CO (11.1 eV; Cox 2000). This is not usually displayed in PDR models, but in Figure 1a we show the atomic and molecular abundances of oxygen for a recent model (Le Bourlot et al. 1993). This model shows the abundances of species in the outer PDR region, as well as abundances in the deep, shielded molecular cloud region, where ion-molecule chemistry dominates. In contrast to $\mathrm{CII}, \mathrm{Or}$ is present in both PDR and shielded molecular cloud regions. OI $(63 \mu \mathrm{m})$ emission would not be observable from deep in the cloud because this region is predicted to be too cold to populate the upper level, but OI may be detectable in absorption if there is a suitable background source. In fact, Poglitsch et al. (1996) reported an absorption feature in the OI spectrum of DR21, taken using the NASA KAO. This feature may be due to a molecular cloud and was taken to indicate a high relative abundance of atomic oxygen in a cold cloud.

The method we have proposed is to examine a range of objects with ISO-LWS (Fabry- Perot mode with resolution of $\sim 35 \mathrm{~km} \mathrm{~s}^{-1}$; Swinyard et al. 1996). Each of the clouds has a strong background continuum source on the line of sight and a different column density of quiescent gas, as seen 
from molecular line data. The final intention is to separate the PDR and quiescent cloud effects by the variation of OI absorption strength with quiescent cloud column density. An initial report of our ISO measurements was given by Keene et al. (1998). The spectrum of Sgr B2 has been found to show very strong OI $(63 \mu \mathrm{m})$ absorption due to several separate absorption clouds, distinguishable by their different velocities, which can be analyzed independently to provide the variation in column density. That forms the subject of this study. An initial report of the Sgr B2 absorption has been made by Baluteau et al. (1997) from a low spectral resolution survey scan using the grating mode of ISO-LWS with resolution of $\sim 1,400 \mathrm{~km} \mathrm{~s}^{-1}$, where the components are not resolved. Baluteau et al. also obtained very useful $\mathrm{N}-\mathrm{S}$ and $\mathrm{E}-\mathrm{W}$ raster scans showing that, off the position of the strong continuum source Sgr B2(M), OI is seen in emission. This emission at $\sim 60 \mathrm{~km} \mathrm{~s}^{-1}$ (Fig. 2) is from Sgr B2 itself, rather than from foreground clouds.

The molecular absorption line clouds in front of Sgr B2 have been studied by a number of authors. Densities are typically found to be in the range $10^{2}-10^{3} \mathrm{~cm}^{-3}$, with some clouds as dense as $2 \times 10^{4} \mathrm{~cm}^{-3}$ (Greaves \& Williams 1994). Temperatures are found to be generally $\$ 15-20 \mathrm{~K}$, except for the -95 to $-105 \mathrm{~km} \mathrm{~s}^{-1}$ component, which is somewhat warmer ( $\sim 35 \mathrm{~K}$; Tieftrunk et al. 1994). These temperatures and densities are much too low to produce significant Or emission. These clouds are rich in molecular species, such as $\mathrm{HCO}^{+}, \mathrm{HCN}, \mathrm{HNC}, \mathrm{CN}, \mathrm{CCH}, \mathrm{C}_{3} \mathrm{H}_{2}, \mathrm{CS}, \mathrm{SiO}$, $\mathrm{N}_{2} \mathrm{H}^{+}, \mathrm{NH}_{3}, \mathrm{CH}_{3} \mathrm{OH}, \mathrm{SO}, \mathrm{H}_{2} \mathrm{~S}$ (Tieftrunk et al. 1994; Greaves \& Nyman 1996) with abundances similar to those observed in dark clouds. Similarly, rich absorption spectra have also been found in diffuse and translucent absorption line clouds in front of extragalactic sources (e.g. Lucas \& Liszt 1997 and references therein). Standard chemical models of diffuse and translucent clouds are unable to reproduce such a rich chemistry, and several alternatives have been proposed, such as turbulent chemistry (Hogerheijde et al. 1995; Joulain et al. 1998) or differences in the elemental depletions and/or the gas phase $[\mathrm{C}] /[\mathrm{O}]$ ratio compared to dark clouds (Turner 2000). Other possibilities include turbulent mixing of different cloud layers (Chièze et al. 1991; Xie et al. 1995), which could enrich the translucent layer by molecules produced in shielded clumps, and also be responsible for the non-appearance of the pure $\mathrm{H}_{2}$ PDR layer (see §3).

\section{Observations}

OI $(63 \mu \mathrm{m})$ observations of Sgr B2 were carried out with both grating and Fabry-Perot modes of ISO-LWS. Figure 2 shows the Fabry-Perot scans for positions Sgr B2(M), and $180^{\prime \prime} \mathrm{N}$ and $180^{\prime \prime}$ $\mathrm{S}$. At the central position absorption is seen for the Sgr $\mathrm{B} 2$ envelope at $60 \mathrm{~km} \mathrm{~s}^{-1}$, as well as the foreground molecular clouds on the line of sight to the Sun at $\sim 0 \mathrm{~km} \mathrm{~s}^{-1}$ and two negative velocities. The observed Fabry-Perot spectra have been deconvolved with a $35 \mathrm{~km} \mathrm{~s}^{-1}$ width Lorentzian representing the LWS resolution function (Swinyard et al. 1996) using a Maximum Entropy Method (MEM) deconvolution algorithm, which results in a factor of $2-3$ improvement in the spectral resolution. Improving the resolution of astronomical images using the MEM is a well established technique in astronomy (see e.g. Narayan \& Nityananada 1986 and references therein), provided the instrumental response function is known. Although usually used for enhancing spatial resolution in images, the technique is perfectly suitable for enhancing spectral resolution as well. It works by finding a model that, when convolved with the instrumental response function, best reproduces the observed spectrum. This is done by modifying the model to minimize $x^{2}$. Depending on the constraints of the image or spectrum, the answer can be ambiguous. In these cases, maximizing the entropy ensures that the model with the least information not required by the data is chosen. This additional constraint is important in cases where little information is present (e.g. with sparse $u v$-coverage in imaging), but becomes less critical for well sampled data. The achievable resolution is difficult to quantify, since it depends on the signal-to-noise ratio. Using these techniques, we developed a simple algorithm to produce a model that, when convolved with the instrumental profile, fits the observed spectrum well. Since our data have high signalto-noise ratio, and they are highly oversampled, we found that the model was unique. To estimate the effect of the MEM deconvolution on the uncertainty of the OI intensities, we added to the observed spectrum random noise with the same rms as the observed spectrum and subsequently 
applied the MEM algorithm to the resulting spectrum. The resulting $1 \sigma$ statistical uncertainties for the intensities of the three OI components are estimated to be $\sim 3-5 \%$.

The Sgr B2 envelope is seen in emission at both offset positions (Fig. 2). However, no emission is seen at the velocities of the foreground clouds. Molecular absorption can indeed be seen at each of the velocities of the Or absorption. Figure 3 compares the ${ }^{13} \mathrm{CO}(1-0)$ absorption spectrum for the Sgr B2(M) line of sight (observed with the IRAM 30-meter telescope) with the LWS OI spectrum. However, the Hi spectrum must also be compared. For Sgr B2(M) there exists such a spectrum (Garwood \& Dickey 1989) and, as can be seen in Figure 3 , $\mathrm{HI}$ absorption is also present for each velocity at which Or absorption is seen.

\section{Discussion}

There are two major complications, which must be dealt with before any OI absorption line can be used to assign atomic oxygen abundance to a molecular cloud. The first is the fact that atomic oxygen will exist in line-of-sight $\mathrm{HI}$ clouds. The clouds, which show HI absorption at the same velocity as ${ }^{13} \mathrm{CO}$, are very likely close to, or attached to the molecular clouds, probably as halos (Wannier et al. 1983; region A in Fig. 1b). This problem can be approached if good quality $\mathrm{HI}$ absorption spectra are available. The second is the abovementioned problem of the PDRs on the line of sight. According to the models, some significant region of the PDR near the surface $\left(\sim 1 A_{v}\right)$ will contain $\mathrm{H}_{2}$ (region $B$ in Fig. 1b), but no $\mathrm{CO}$ or other trace molecules whose presence defines the molecular cloud (region C). Region B, like the $\mathrm{HI}$ region, will contain OI, and should also contribute to the absorption. ${ }^{4}$ The assignment of OI to the molecular cloud is therefore a tricky operation. Although various PDR models show differences in the depth into the cloud of the $\mathrm{HI} / \mathrm{H}_{2}$ interface, they all have an $\mathrm{H}_{2}$ region bereft of $\mathrm{CO}$ (region $\mathrm{B}$ ) of roughly the same extent.

We may wonder if $\mathrm{O}_{1}$ in the PDR (region B)

\footnotetext{
${ }^{4}$ It is important to note that the density of these PDRs is likely to be quite low (a few $10^{2}-$ a few $10^{3} \mathrm{~cm}^{-3}$ ), so that $\mathrm{Ol}_{1}$ atoms are all in the ground state and the $\mathrm{Ol}_{1}$ column density is accurately determined by an absorption measurement.
}

will be detectable. From the model there is $\sim 1 \mathrm{~A}_{\mathrm{v}}$ of such material on the line of sight through a molecular cloud. This would correspond to a detectable $\mathrm{O}$ absorption with an equivalent width of $3.5 \mathrm{~km} \mathrm{~s}^{-1}$, assuming an $\mathrm{O} / \mathrm{H}$ ratio of $3.2 \times 10^{-4}$. However, aside from the problem of not being able to identify the purely $\mathrm{Cl}$ region (Keene et al. 1985), there is another measurement which raises doubt about the existence of a pure $\mathrm{H}_{2}$ region. The modeling by Andersson and Wannier (1993) of observations of dark clouds in the lines of $\mathrm{HI}, \mathrm{OH}$, and $\mathrm{CO}$ leads to their interpretation that $\mathrm{HI}$ halos lie much closer to the molecular clouds as defined by $\mathrm{CO}$ observations than predicted by standard chemical and photo-dissociation models as of that date. They conclude that the formation rate of $\mathrm{H}_{2}$ used in the models is probably too large, leading to a region of pure $\mathrm{H}_{2}$ much larger than is observed.

Thus, the method here, to compare Or absorptions after subtracting the contribution from the $\mathrm{HI}$ halo in clouds of varying $\mathrm{CO}$ column density, should allow the identification of any surface region, by means of the Or intercept. If there is a proportionality found between $\mathrm{OI}$ and $\mathrm{CO}$, this will provide the relative abundance of atomic oxygen in $\mathrm{CO}$ regions.

Referring to the MEM-deconvolved OI spectrum of Sgr B2 (Fig. 2), we see that the spectrum naturally separates into three velocity ranges corresponding to absorption by foreground clouds at velocities $<-78 \mathrm{~km} \mathrm{~s}^{-1},-78$ to $-25 \mathrm{~km} \mathrm{~s}^{-1}$, and -25 to $30 \mathrm{~km} \mathrm{~s}^{-1}$, plus the absorption feature at $\sim 60 \mathrm{~km} \mathrm{~s}^{-1}$ corresponding to the envelope of Sgr B2 itself. In this paper we are not concerned with the Sgr B2 envelope feature at $60 \mathrm{~km} \mathrm{~s}^{-1}$, because it is likely to be contaminated by OI emission, but will analyze the foreground clouds. For these three velocity ranges we have $\mathrm{OI}, \mathrm{HI}$, and ${ }^{13} \mathrm{CO}$ spectra, which allow us to determine the atomic oxygen, atomic hydrogen, and ${ }^{13} \mathrm{CO}$ column densities for the three Or velocity components (Table 1). From the high spectral resolution ${ }^{13} \mathrm{CO}$ and $\mathrm{HJ}$ spectra it is plausible that the three OI absorption features further break into multiple components (see Fig. 3).

Since we have the HI column density for each velocity range, using the measured $[\mathrm{O}] /[\mathrm{H}]$ ratio in diffuse clouds (3.2 $\times 10^{-4}$; Meyer et al. 1998) we can determine the OI column density associated with the HI region and subtract this from the ob- 
served OI column density, $N(\mathrm{OI})_{\text {obs }}$, to get the $\mathrm{OI}$ column density associated with the molecular $\mathrm{H}_{2}$ gas, $N(\mathrm{OI})_{\mathrm{mol}}$. I.e., whatever is left must be associated with the PDR $\mathrm{H}_{2}$ region (region $\mathrm{B}$ in Fig. 1b) plus the ${ }^{13} \mathrm{CO}$ region (region $\mathrm{C}$ in Fig. 1b). However, in any individual case it is not possible to separate the two contributions to $N(\mathrm{Ol})_{\mathrm{mol}}$ and therefore not possible to claim a knowledge of the atomic oxygen content of molecular clouds as defined by ${ }^{13} \mathrm{CO}$. In principle we need to know the number of PDRs on the line of sight for a given velocity range in order to compare the total ${ }^{13} \mathrm{CO}$ column density with OI for that range, or alternatively by comparing mean column density per ${ }^{13} \mathrm{CO}$ component with mean Or column density over that range. The $\mathrm{OI}$ and ${ }^{13} \mathrm{CO}$ column densities are given in Table 1, together with the number of ${ }^{13} \mathrm{CO}$ components in each range.

As a simplest approximation we assume that the PDRs are all the same and that there is a universal $[\mathrm{OI}] /\left[{ }^{13} \mathrm{CO}\right]$ coefficient, $X$. Then, for each velocity range

$$
\begin{gathered}
N(\mathrm{OI})_{\mathrm{mol}} / n_{\text {comp }}=N(\mathrm{OI})_{\mathrm{H}_{2} / \mathrm{PDR}}+ \\
X N\left({ }^{13} \mathrm{CO}\right) / n_{\text {comp }}
\end{gathered}
$$

Since we have three ranges, $N(\mathrm{OI})_{\mathrm{H}_{2} / \mathrm{PDR}}$ and $X$ can be determined from the least squares line fit. The plot of $N(\mathrm{OI})_{\mathrm{mol}} / n_{\text {comp }}$ vs. $N\left({ }^{13} \mathrm{CO}\right) / n_{\text {comp }}$ is shown in Figure 4. From this plot it is inferred that there is indeed a reasonably constant relation between $\mathrm{OI}$ and ${ }^{13} \mathrm{CO}$ column densities and that $[\mathrm{OI}] /\left[{ }^{13} \mathrm{CO}\right]$ is $270 \pm 35$ ( $1 \sigma$ uncertainty for the slope based on the statistical uncertainties in ${ }^{13} \mathrm{CO}$ and Ol column densities). After adding in quadrature $15 \%$ calibration uncertainties for ${ }^{13} \mathrm{CO}$ and OI to the $3 \sigma$ statistical uncertainty for the slope, we obtain $[\mathrm{OI}] /\left[{ }^{13} \mathrm{CO}\right]=270 \pm 120(3 \sigma$; not including modeling uncertainties) for the foreground clouds on the line of sight toward Sgr B2(M). Assuming a ${ }^{13} \mathrm{CO}$ abundance of $2 \times 10^{-6}$ relative to $\mathrm{H}_{2}$ (Dickman 1978$)^{5}$ leads to an atomic oxygen abundance of $(2.7 \pm 1.2) \times 10^{-4}$ relative to

\footnotetext{
${ }^{5}$ The ${ }^{13} \mathrm{CO}$ abundace derived by Dickman (1978) corresponds to local dark clouds. One might expect the ${ }^{13} \mathrm{CO}$ abundance to increase with decreasing galactocentric distance following the observed variation in the $\mathrm{CO} /{ }^{13} \mathrm{CO}$ abundance ratio (Langer \& Penzias 1990). However, Lis \& Goldsmith (1989) derived a ${ }^{13} \mathrm{CO}$ abundance of $1 \times 10^{-6}$ relative to $\mathrm{H}_{2}$ in the envelope of the $\mathrm{Sgr} \mathrm{B} 2$ molecular cloud, a factor of 2 lower than the local value. We thus use the lo-
}

$H$ in the foreground molecular clouds, comparable to the value found in diffuse clouds. The deduced $[\mathrm{OI}] /[\mathrm{CO}]$ value for the molecular gas is $\sim 9 \pm 4(3 \sigma)$ for a $[\mathrm{CO}] /\left[{ }^{13} \mathrm{CO}\right]$ abundance ratio of 30 (Langer \& Penzias 1990). Also from the plot it appears that there is no nonzero intercept, $(-1.1 \pm 4.6) \times 10^{16} \mathrm{~cm}^{-2}$, (a $1 \sigma$ statistical uncertainty, corresponding to $A_{v}=0.08$, assuming the diffuse cloud Or abundance) so, within the uncertainties, there is no manifestation of a PDR $\mathrm{H}_{2}$ region devoid of ${ }^{13} \mathrm{CO}$. Incidentally, the fact that this region is not seen makes the validity of the assumption of similar PDRs moot.

The formula given in eq. (1) corresponds to a physical model with a number of ${ }^{13} \mathrm{CO}$ clumps distributed along the line of sight, each one exposed to the external UV field and therefore surrounded by its own PDR. The other extreme is a collection of clumps packed closely together with only external surfaces exposed to the UV field (one common PDR for all clumps; $n_{\text {comp }}=1$ ). In this case, a least squares fit gives an $[\mathrm{OI}] /\left[{ }^{13} \mathrm{CO}\right]$ ratio of $290 \pm 24(1 \sigma)$, statistically consistent with that derived above, and again no nonzero intercept. Our results are thus insensitive to the exact number of ${ }^{13} \mathrm{CO}$ components.

\section{Conclusion}

We have presented a high signal-to-noise ratio ISO-LWS Fabry-Perot spectrum of the OI $(63 \mu \mathrm{m})$ absorption toward Sgr B2, which is of sufficient quality to permit deconvolution of the instrument spectral function, thus revealing four separate velocity ranges of absorption. This spectrum correlates well with both the $\mathrm{HI}$ absorption spectrum and the ${ }^{13} \mathrm{CO}$ absorption spectrum. In order to determine the atomic oxygen abundance associated with the molecular clouds, as defined by ${ }^{13} \mathrm{CO}$, we first subtract the OI absorption components, deduced from the $\mathrm{HI}$ spectrum, for the three velocity ranges corresponding to foreground clouds on the line of sight toward Sgr B2. We then compute the oxygen column density remaining and correlate that with the ${ }^{13} \mathrm{CO}$ column density per cloud component as found from the number of distinct ${ }^{13} \mathrm{CO}$ velocity absorption features in each of the three $\mathrm{OI}$

cal value of $2 \times 10^{-6}$ as an average for the clouds on the line of sight toward Sgr B2. This value is uncertain by about a factor of 2 . 
absorption ranges. The plot shows a good fit to a constant [Or] $/\left[{ }^{13} \mathrm{CO}\right]$ ratio of $270 \pm 120(3 \sigma)$ with no apparent intercept. This implies that there is indeed a large atomic oxygen content in molecular clouds (Fig 1b, region C), but there is no evidence for atomic oxygen in the $\mathrm{H}_{2}$ region devoid of $\mathrm{CO}$ predicted by PDR models. The probable reason for the absence of the model predicted region $B$ is the inhomogeneous nature of the cloud surface, which effectively mixes the various surface regions.

The result then depends on how we describe a molecular cloud. If we define the clouds as filling the size scale of the pencil beam to the source, the OI is correlated with ${ }^{13} \mathrm{CO}$ and it is reasonable to say that atomic oxygen is present in the $\mathrm{CO}$ containing clouds. If we define a cloud as one of the small clumps presumed to be part of the inhomogeneous structure of the big cloud, it has not been proved that atomic oxygen spatially coexists with the $\mathrm{CO}$, as it may be in a surface $\mathrm{H}_{2}$ region. The result may be best stated that, observationally, there is an apparent correlation of $[\mathrm{OI}] /[\mathrm{CO}] \simeq 9$ in the ISM molecular clouds on the line of sight toward Sgr B2. We find a much lower [OI]/[CO] ratio in the molecular gas than recently reported in L1689N ( 50; Caux et al. 1999). Our result is also a little lower than that found for the cold clouds on the line of sight toward W49N ( 15; Vastel et al. 2000).

The observed $[\mathrm{Or}] /\left[{ }^{13} \mathrm{CO}\right]$ column density ratio of $270 \pm 120$ can be easily explained in the framework of PDR models of low density clouds (Fig. 6, solid line; $n_{\mathrm{H}}=100 \mathrm{~cm}^{-3}, G_{0}=1$ ), or high density couds illuminated by a strong UV field (Fig. 6 , dashed line; $\left.n_{\mathrm{H}}=10^{4} \mathrm{~cm}^{-3}, G_{0}=100\right)$. The PDR model predicts a lower $[\mathrm{OI}] /\left[{ }^{13} \mathrm{CO}\right]$ ratio for high density clouds illuminated by a weak $U V$ field (Fig. 6, dotted line; $n_{\mathrm{H}}=10^{4} \mathrm{~cm}^{-3}, G_{0}=1$ ), except for diffuse regions $\left(A_{v}<1\right)$. However, the model does not take into account $\mathrm{CO}$ depletion onto dust grains in high-density, low-temperature regions that is likely to be important for highcolumn density clouds, such as those studied by Caux et al. (1999) and Vastel et al. (2000) (see e.g. Kramer et al. 1999, Caselli et al. 1999).

This research is based on observations with ISO, an ESA project with instruments funded by ESA Member States with the participation of ISAS and NASA, and has been supported by
NASA under ISO Block Grant to the California Institute of Technology and by NSF grant AST9980846 to the Caltech Submillimeter Observatory. We thank J. Le Bourlot, G. Pineau des Forêts and $E$. Roueff for the permission to use their PDR model.

\section{REFERENCES}

Andersson, B.-G. \& Wannier, P. G. 1993, ApJ, 402,585

Bergin, E. A., Melnick, G. J., Stauffer, J. R., et al. 2000, ApJ, 539, 129

Baluteau, J.-P., Cox, P., Cernicharo, J. et al. 1997, A\&A, 322, L23

Cardelli, J.A., Ebbets, D.C., \& Savage, B.D. 1993, ApJ, 413, 401

Caselli, P., Walmsley, C.M., Tafalla, M., Dore, L., Myers, P.C. 1999, ApJ, 523, L165

Caux, E., Ceccarelli, C., Castets, A., et al. 2000, $A \& A, 347$, L1

Cernicharo, J., Lim, T., Cox, P., GonzalezAlfonso, E., Caux, E., Swinyard, B.M., MartínPintado, J., Baluteau, J. P., \& Clegg, P. 1997, A\&A, 323, L25

Chiéze, J.-P., Pineau des Forêts, G., \& Herbst, E. ApJ, 1991, 373, 110

Cohen, R.J. 1977, MNRAS, 178, 547

Cox, A.N., er. 2000, Allen's Astrophysical Quantities (4th ed.; New York: Springer)

Dalgarno, A., \& Black, J.H. 1976, RPPh, 39, 573

Dickman, R.L. 1978, ApJS, 37, 407

Fuente, A., Cernicharo, J., Garcia-Burillo, S., \& Tejero, J. 1993, A\&A, 275, 558

Garwood, R.W., \& Dickey, J.M. 1989, ApJ,338, 841

Goldsmith, P. F., Melnick, G. J., \& Bergin, E. A. et al. 2000, ApJ, 539, L123

Greaves, J.S., \& Nyman, L.-A. 1996, A\&A, 305, 950 
Greaves, J.S., \& Williams, P.G. 1994, A\&A, 290, 259

Herbst, E., \& Klemperer, W. 1973, ApJ, 185, 505

Hogerheijde, M. R., de Geus, E. J.,Spaans, M., van Langevelde, H. J., \& van Dishoeck, E. F. 1995, ApJ, 441, L93

Hollenbach, D.J., Takahashi, T., \& Tielens, A.G.G.M. 1991, ApJ, 377, 192

Hollenbach, D. J., \& Tielens, A.G.G.M. 1999, RevMP, 71,173

Jacq, T., Henkel, C., Walmsley, C.M., Jewell, P.R., \& Baudry, A. 1988, A\&A, 199, L5

Joulain, K., Falgarone, E., Pineau des Forêts, G., Flower, D.R. 1998, A\&A, 340, 241

Keene, J., Blake, G.A., Phillips, T.G., Huggins, P.J., Beichman, C.A. 1985, ApJ, 299, 697

Keene, J., Lis, D.C.., Phillips, T.G., \& Schilke, P. 1997, in Molecules in Astrophysics: Probes and Processes, ed. E. van Dishoeck (Kluwer), 129

Knacke, R.F., \& Larson, H.P. 1991, ApJ, 367, 162

Kramer, C., Alves, J., Lada, C.J., Lada, E.A., Sievers, A., Ungerechts, H., \& Walmsley, C.M. 1999, A\&A, 342, 257

Lada, C.J., Lada, E.A., Clemens, D.P., \& Bally, J. 1994, ApJ, 429, 694

Langer, W. 1976, ApJ, 206, 699

Langer, W.D., \& Penzias, A.A. 1990, ApJ, 357, 477

Le Bourlot, J., Pineau des Forêts, G., Roueff, E., \& Flower, D.R. 1993, A\&A, 267, 233

Lis, D.C., \& Goldsmith, P.F. 1989, ApJ, 337, 704

Liszt, H. S., Vanden Bout, P. A. 1985, ApJ, 291, 178

Lucas, R., \& Liszt, H. 1997, in CO: Twenty-Five Years of Millimeter-Wave Spectroscopy, eds. W. Latter et al. (Kluwer: Dordrecht), 95

Melnick, G. J., Ashby, M.L.N., Plume, R. et al. 2000, ApJ, 539, L87
Melnick, G., Gull, G. E., Harwit, M. 1979, ApJ, 227, L29

Meyer, D.M., Jura, M., Cardelli, J.A. 1998, ApJ, 493, 222

Naraya, R., \& Nityanananda, R. 1986, ARAA, 24, 127

Poglitsch, A., Herrmann, F., Genzel, R.; Madden, S.C., Nikola, T., Timmermann, R., Geis, N., Stacey, G.J. 1996, ApJ, 462, L43

Phillips, T. G., van Dishoeck, E.F., Keene, J. 1992, ApJ, 399, 533

Phillips, T.G., \& Huggins, P.J. 1981, ApJ, 251, 533

Russell, R.W., Melnick, G., Gull, G.E., \& Harwit, M. 1980, ApJ, 240, L99

Snell, R. L., Howe, J. E., Ashby, M.L.N. et al. 2000a, ApJ, 539, L93

Snell, R. L.; Howe, J. E.; Ashby, M.L.N. et al. 2000b, ApJ, 539, L101

Snow, T.P., Black, J.H., van Dishoeck, E.F., Burks, G., Crutcher, R.M., Lutz, B.L., Hanson, M.M., \& Shuping, R.Y. 1996, ApJ, 465, 245

Swinyard, B.M., Clegg, P.E. et al. 1996, A\&A, $315, \mathrm{~L} 43$

Tieftrunk, A., Pineau des Forêts, G., Schilke, P., \& Walmsley, C.M. 1994, 289, 579

Turner, B.E. 2000, ApJ, 542, 837

van Dishoeck, E.F., Blake, G.A., Draine, B.T., \& Lunine, J. I. 1993, in Protostars and Planets III, eds. E. Leby \& J. Lunine (U. Arizona Press: Tucson), 163

Vastel, C., Caux, E., Ceccareli, C., Castets, A., Gry, C., \& Baluteau, J.P. 1999, A\&A, 357, 994

Wannier, P.G., Lichten, S.M., \& Morris, M. 1983, ApJ, 268, 727

Whittet, D.C.B. 1992, Dust in the Galactic Environment (Bristol: A. Hilger), 158

Xie, T., Allen, M., \& Langer, W. D. 1995, ApJ, 440,674 
Zmuidzinas, J., Blake, G.A., Carlstrom, J., Keene, J., Miller, D., Schilke, P., \& Ugras, N.G. 1995, Airborne Astronomy Symposium on the Galactic Ecosystem: From Gas to Stars to Dust, eds. M. Haas et al. (ASP Conf. Ser. Vol. 73), 33

\section{FIGURE CAPTIONS}

Fig. 1.- (a) PDR abundances relative to $H$ as a function of depth into the cloud, after Le Bourlot et al. (1993). The model cloud has a volume density $n\left(\mathrm{HI}+2 \mathrm{H}_{2}\right)=10^{4} \mathrm{~cm}^{-3}$ (toward the high end of the values derived by Greaves \& Williams 1994, Greaves \& Nyman 1996, and Lucas \& Liszt 1997), and the UV field intensity $G_{o}=1$ (standard interstellar radiation field). The total oxygen and carbon abundances are $3 \times 10^{-4}$ and $1.5 \times 10^{-4}$, respectively. Note that $\mathrm{O}_{2}$ and $\mathrm{H}_{2} \mathrm{O}$ are minor species in the model and oxygen is primarily in atomic form and in $\mathrm{CO}$ in the molecular region. The $\mathrm{CO}$ abundance in the cloud interior decreases with decreasing gas density (owing to the decreased $\mathrm{CO}$ formation rate) and increasing UV field intensity (owing to the increased CO photodissociation rate). (b) Overall geometry of $\mathrm{HI}$, $\mathrm{H}_{2}$, and $\mathrm{CO}$ regions in a PDR (from Hollenbach \& Tielens 1999; the region designations A, B, and $\mathrm{C}$ are added here).

Fig. 2.- OI $(63 \mu \mathrm{m})$ Fabry-Perot spectra toward Sgr B2(M) (upper panel), 180" N, and 180" $\mathrm{S}$ (lower-panel, upper and lower curves, respectively). Black and gray lines correspond to the raw and MEM-deconvolved spectra, respectively. The lower-panel vertical axes on the left and right correspond to the $180^{\prime \prime} \mathrm{N}$ and $180^{\prime \prime} \mathrm{S}$ spectra, respectively. The intensity scale is in the LWS FabryPerot units as given by the LWS analysis package (ISAP).

Fig. 3.- (Upper panel) Observed OI $(63 \mu \mathrm{m})$ Fabry-Perot spectrum $\left(\sim 35 \mathrm{~km} \mathrm{~s}^{-1}\right.$ resolution, gray line) and MEM-deconvolved spectrum ( 10 $15 \mathrm{~km} \mathrm{~s}^{-1}$ resolution, solid black line). (Lower panel) $\mathrm{HI}$ and ${ }^{13} \mathrm{CO}(1-0)$ absorption spectra toward Sgr B2(M) (gray and black lines, respectively). The ${ }^{13} \mathrm{CO}$ spectrum toward the central

This 2-column preprint was prepared with the AAS LATEX macros v5.0. position has been corrected for the cloud emission averaged over a $40^{\prime \prime}$ square ring.

Fig. 4. - OI column density as a function of ${ }^{13} \mathrm{CO}$ column density per ${ }^{13} \mathrm{CO}$ velocity component for the three velocity ranges that are distinguishable in the MEM-deconvolved OI spectrum. Errorbars correspond to $1 \sigma$ statistical uncertainties for $\mathrm{OI}$ and ${ }^{13} \mathrm{CO}$ column densities. A least squares fit to the data gives a slope of $270 \pm 35$ ( $1 \sigma$ statistical uncertainty). The intercept is $(-1.1 \pm 4.6) \times 10^{16}$ $\mathrm{cm}^{-2}(1 \sigma)$. This indicates that the $\mathrm{OI}$ and ${ }^{13} \mathrm{CO}$ emission come from the same region and there is no excess OI emission from the PDR interface where hydrogen is already molecular, but ${ }^{13} \mathrm{CO}$ is photodissociated (region B in Fig. 1b).

Fig. 5.- Observed and model OI $(63 \mathrm{~m})$ absorption spectra (black and gray curves, respectively), assuming the $\mathrm{OI}$ abundance of $3.2 \times 10^{-4}$ in the $\mathrm{HI}$ region and $2.6 \times 10^{-4}$ in the molecular gas (20\% depletion) at raw LWS resolution $\left(\sim 35 \mathrm{~km} \mathrm{~s}^{-1}\right.$; upper panel) and the deconvolved resolution $(\sim 10$ $15 \mathrm{~km} \mathrm{~s}^{-1}$; lower panel). The part of the spectrum at VLSR $\gtrsim 30 \mathrm{~km} \mathrm{~s}^{-1}$ is contaminated by the emission form the Sgr B2 envelope and is not considered here. The discrepancies between the observed and model spectrum at the deconvolved resolution (lower panel) may indicate variations in the $\mathrm{OI}$ to $\mathrm{CO}$ abundance ratio between different components. In addition, given the broad wings of the instrumental profile, the Ol optical depth and column density for the $0 \mathrm{~km} \mathrm{~s}^{-1}$ component may be underestimated owing to the contamination by OI emission from the Sgr B2 envelope.

Fig. 6.- OI to ${ }^{13} \mathrm{CO}$ column density ratio from the cloud surface as a function of visual extinction for PDR models with varying gas densities and UV field intensities. The shaded area outlines the ratio derived for the clouds along the line of sight toward Sgr B2. 
Table 1: Column densities of $\mathrm{HI}, \mathrm{OI}$, and ${ }^{13} \mathrm{CO}$ in the three Or velocity ranges.

\begin{tabular}{cccccccc}
\hline Velocity & $N(\mathrm{HI})$ & $N(\mathrm{OI})_{\text {obs }}$ & $N(\mathrm{OI})_{\text {mol }}$ & $N\left({ }^{13} \mathrm{CO}\right)$ & $\mathrm{OI} /{ }^{13} \mathrm{CO}$ & $n_{\text {comp }}$ & $\mathrm{A}_{\mathrm{v}}$ \\
\hline$v<-78$ & $7.7 \times 10^{20}$ & $2.5 \times 10^{18}$ & $2.3 \times 10^{18}$ & $9.5 \times 10^{15}$ & 240 & 9 & 5 \\
$-78<v<-25$ & $2.6 \times 10^{21}$ & $6.4 \times 10^{18}$ & $5.6 \times 10^{18}$ & $2.2 \times 10^{16}$ & 260 & 11 & 12 \\
$-25<v<30$ & $1.2 \times 10^{22}$ & $9.6 \times 10^{18}$ & $5.6 \times 10^{18}$ & $2.0 \times 10^{16}$ & 280 & 17 & 11 \\
\hline
\end{tabular}

NoTES: Entries in the table are: velocity range $\left(\mathrm{km} \mathrm{s}^{-1}\right)$, Hi column density $\left(\mathrm{cm}^{-2}\right)$, OI column density $\left(\mathrm{cm}^{-2}\right)$, Or column density in the molecular gas $\left(\mathrm{cm}^{-2}\right),{ }^{13} \mathrm{CO}$ column density $\left(\mathrm{cm}^{-2}\right),[\mathrm{OI}] /\left[{ }^{13} \mathrm{CO}\right]$ abundance ratio in the molecular gas, the number of ${ }^{13} \mathrm{CO}$ components, and the total visual extinction per OI velocity range computed assuming a ${ }^{13} \mathrm{CO}$ abundance of $1 \times 10^{-6}$ with respect to $\mathrm{H}$ nuclei. $\mathrm{HI}$ column densities have been computed using the formulae and spin temperatures of Cohen (1977) and ${ }^{13} \mathrm{CO}$ column densities are based on LVG modeling of the ${ }^{13} \mathrm{CO}(1-0)$ absorption spectrum (Fig. 3, lower panel). 
Figure 1a

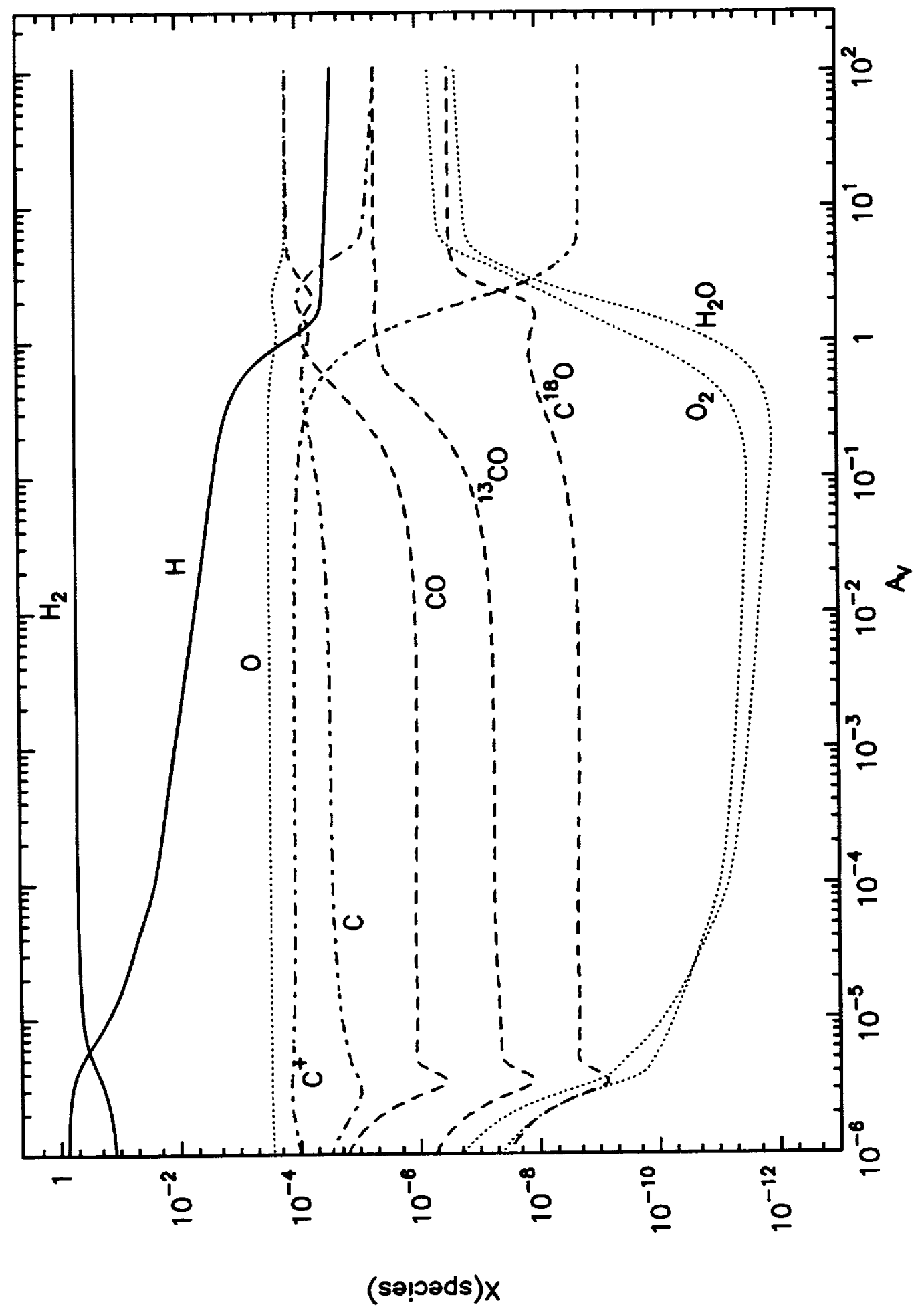


Figure 1b

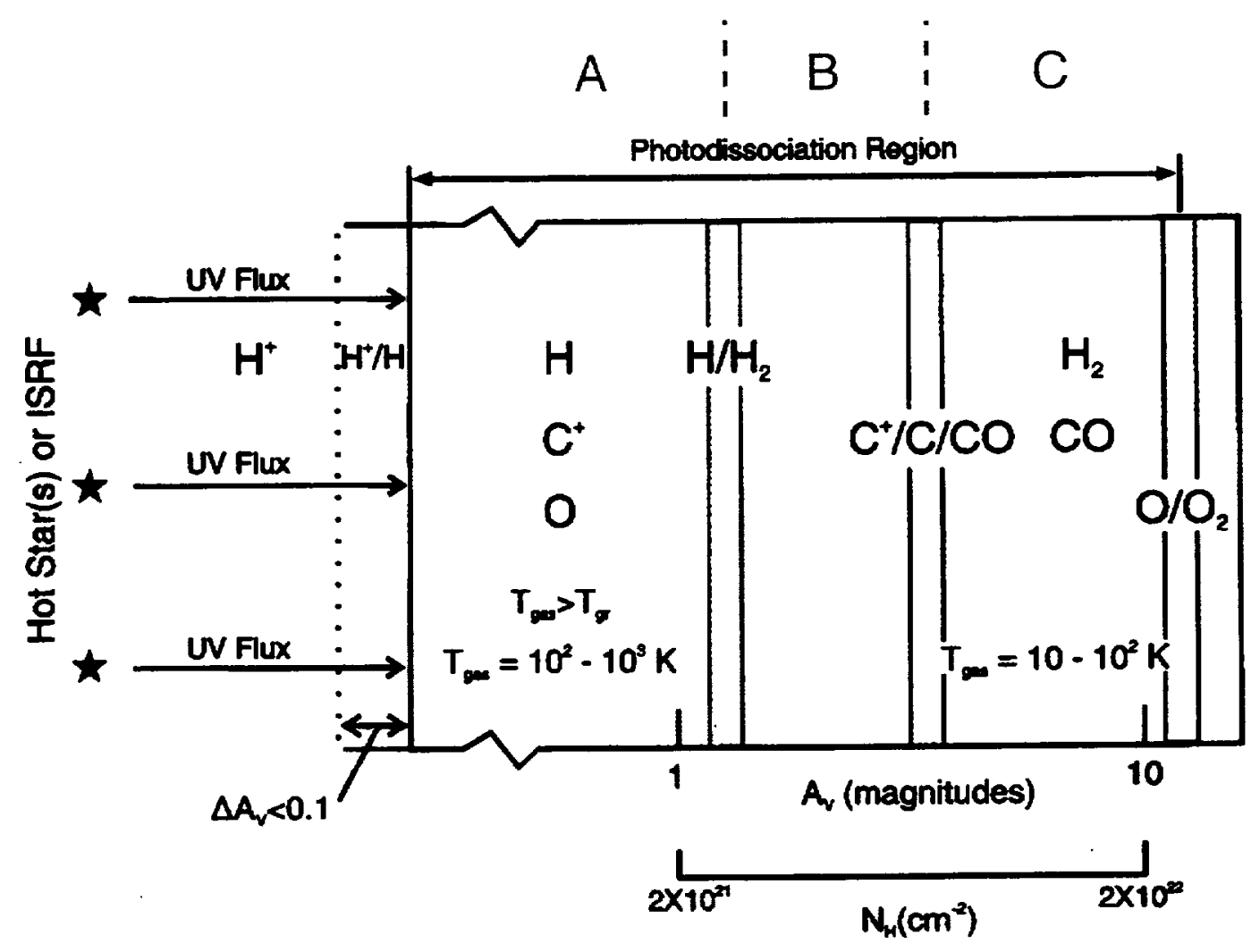


Figure 2

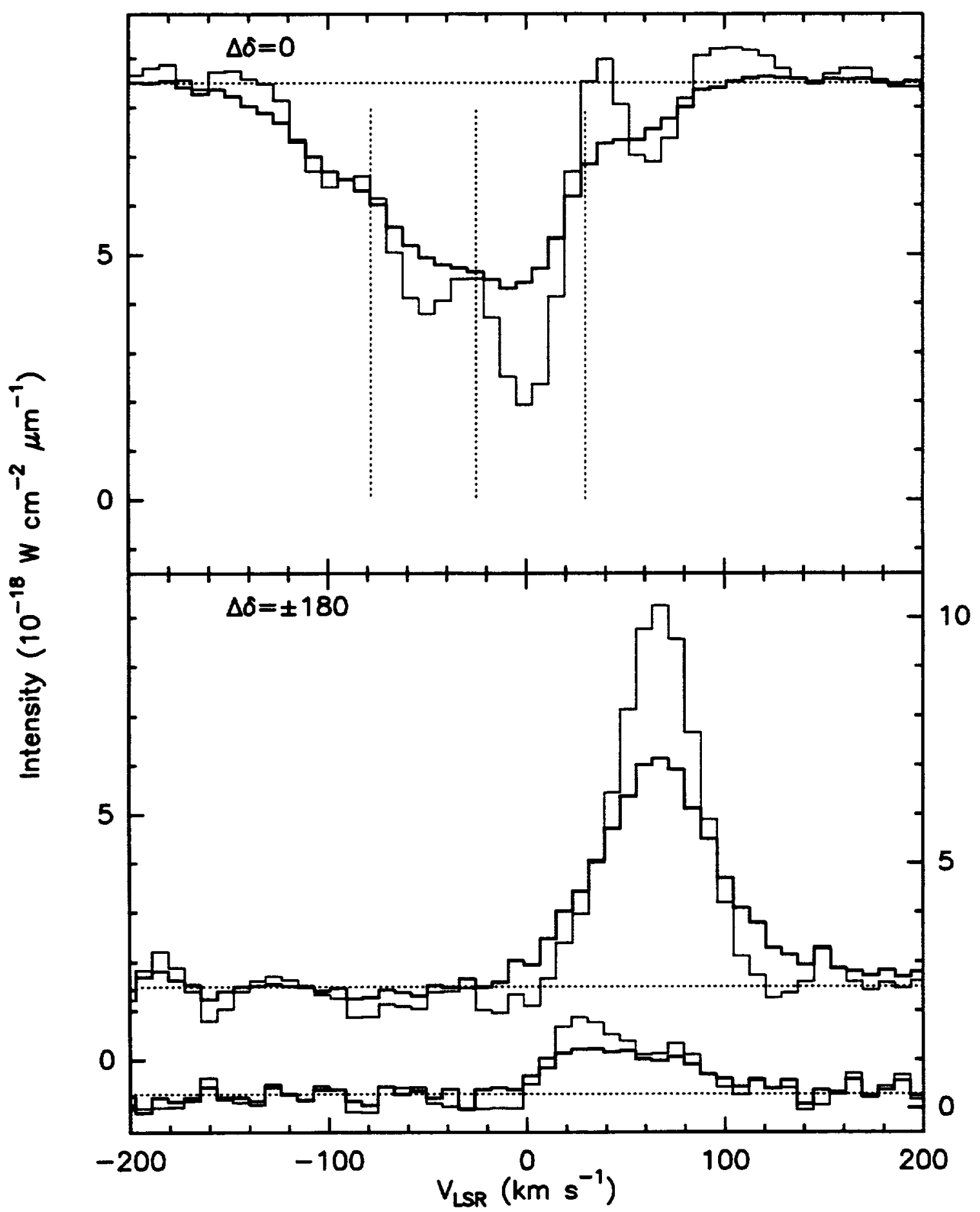


Figure 3

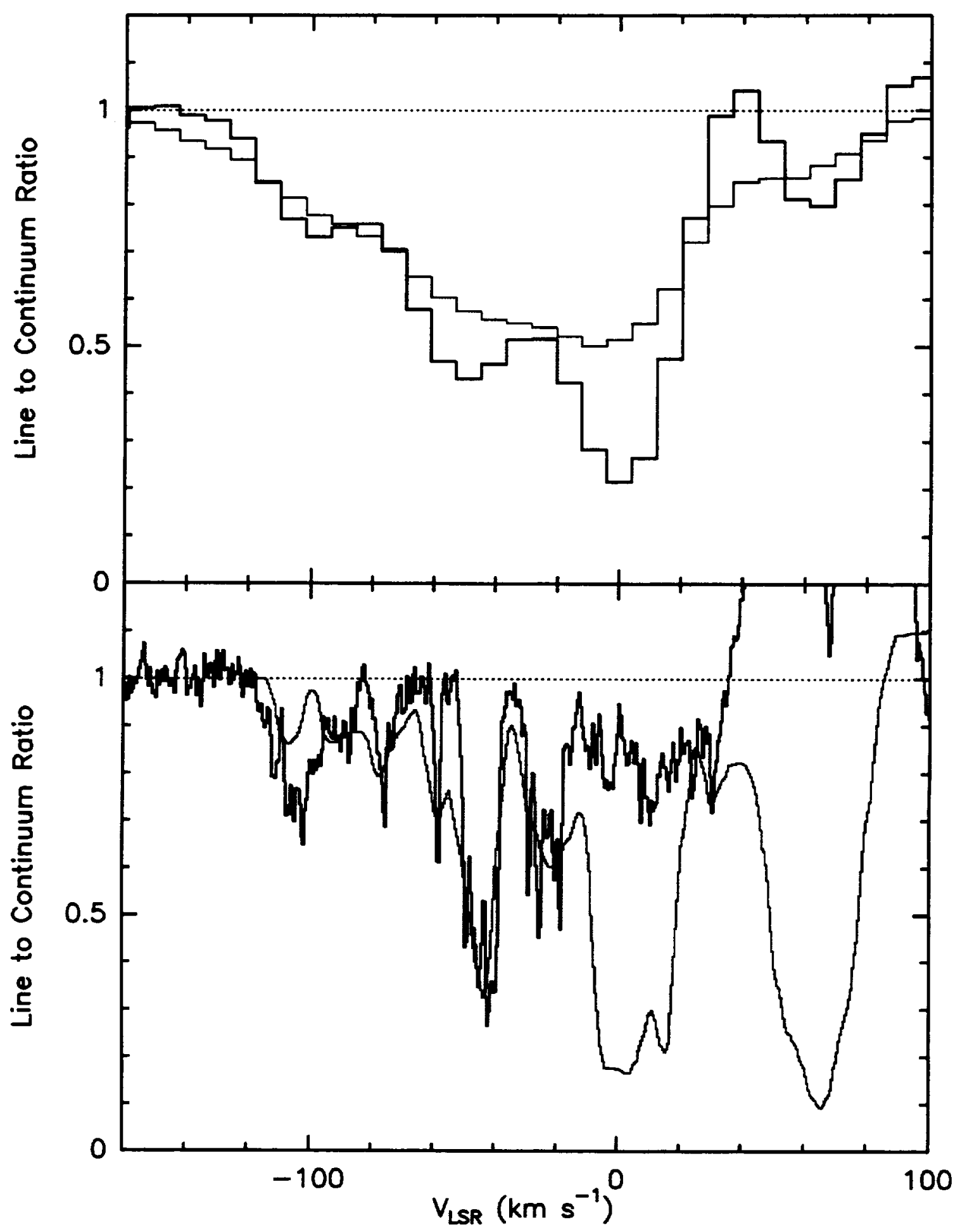


Figure 4

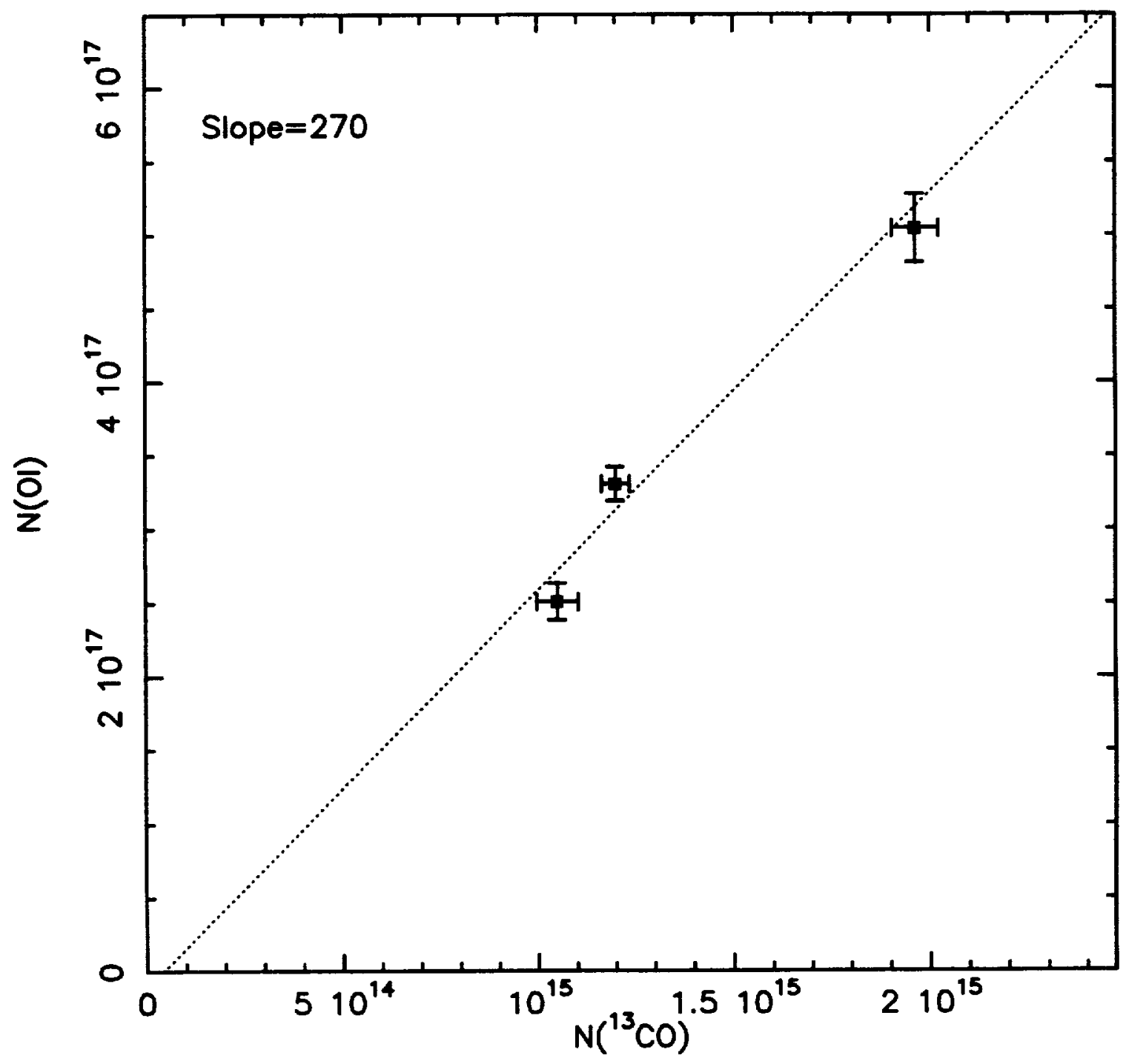


Figure 5

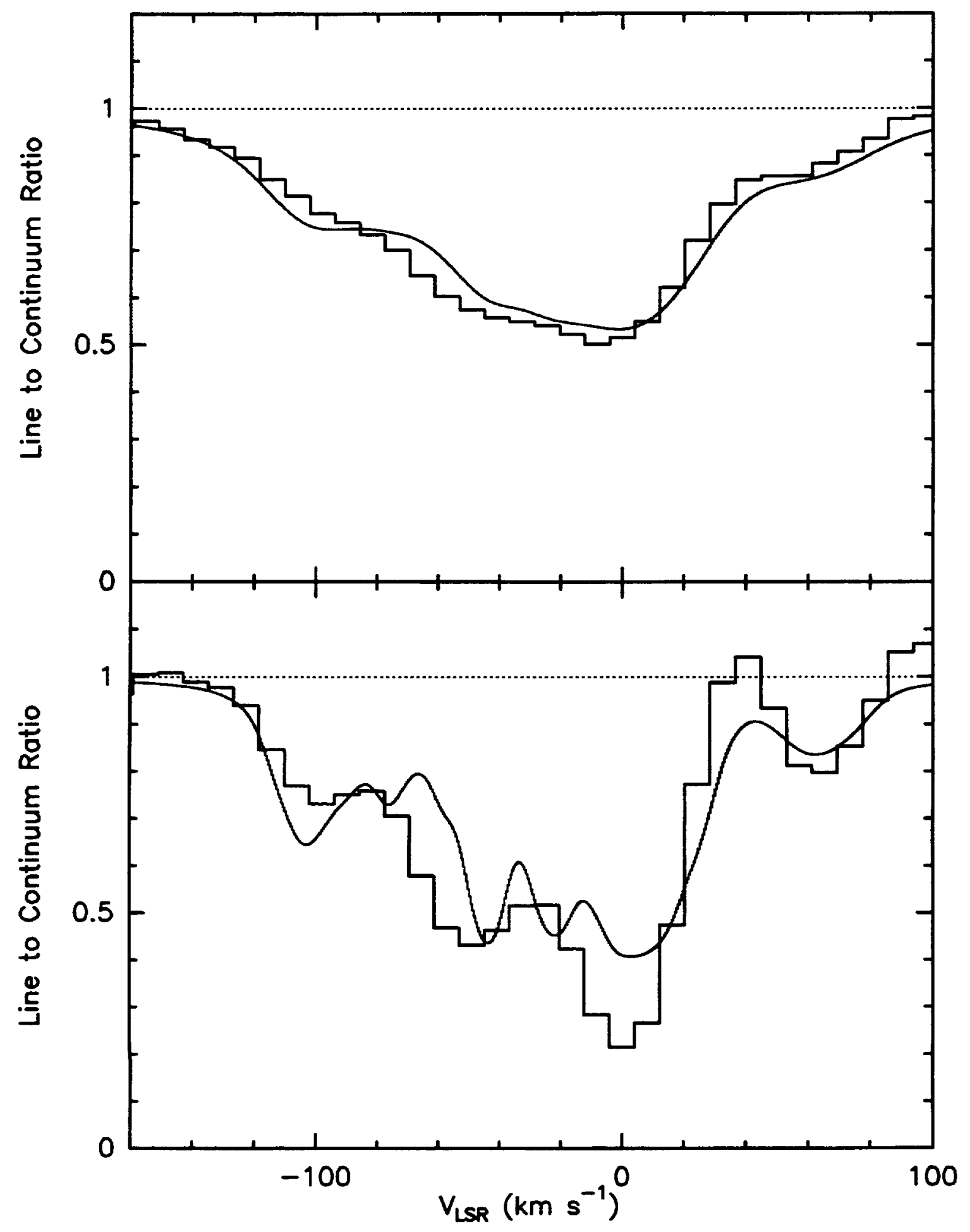


Figure 6

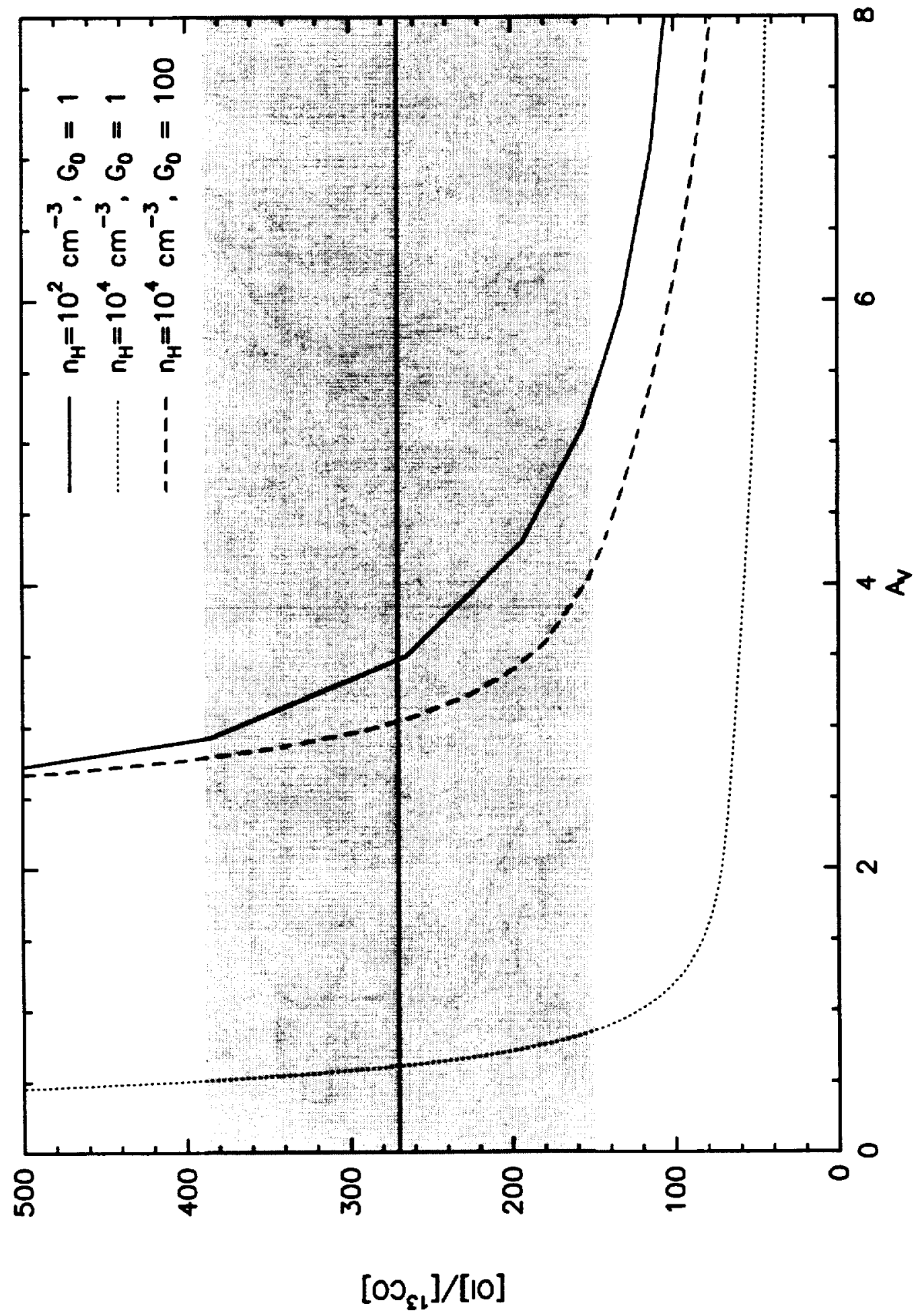

